\title{
Prospective Study of the Effectiveness of Coping in Pediatric Patients
}

\author{
Daniel Zehnder, MA \\ Alice Prchal, MA \\ Department of Psychosomatics and Psychiatry, University Children's \\ Hospital Zurich, Switzerland \\ Margarete Vollrath, PhD \\ Division of Mental Health, Norwegian Institute of Public Health, Oslo, \\ Norway \\ Markus A. Landolt, PhD \\ Department of Psychosomatics and Psychiatry, University Children's \\ Hospital Zurich, Switzerland
}

\begin{abstract}
Findings about the influence of coping on psychological adjustment in children with different medical conditions are inconsistent and often based on cross-sectional data. This prospective study evaluated the effect of various coping strategies on children's post-traumatic stress symptoms and behavioral problems 1 month and 1year after an accidental injury or the diagnosis of a chronic disease in 161 pediatric patients 6-15 years of age. Only minor positive effects of coping on psychosocial adjustment were found: Religious coping reduced post-traumatic stress symptoms. Active coping strategies had negative effects on internalizing and externalizing behavioral problems. Support seeking strategies, distraction, and avoidance had no impact on long-term psychosocial adjustment.
\end{abstract}

KEY WORDS: coping; chronic disease; injury; adjustment; post-traumatic stress disorder; behavior.

\section{Introduction}

Pediatric patients are confronted with different medical conditions and a variety of stressful situations that require coping responses. Conceptually, coping is viewed as a mediator between a stressor and the outcome of exposure to that stressor, and it is defined as constantly changing cognitive and behavioral efforts to manage

Address correspondence to Markus A. Landolt, PhD, University Children's Hospital Zurich, Steinwiesstrasse 75, CH-8032 Zurich, Switzerland; e-mail: markus.landolt@kispi.unizh.ch. 
specific demands that are appraised as taxing or exceeding personal resources. ${ }^{1}$ There are many classifications of coping efforts, which typically, are categorized bipolarly. ${ }^{2}$ An example of a common classification is problem-focused coping that aims at resolving or changing the stressful situation, and emotion-focused coping to manage emotional reactions to the situation. ${ }^{1}$

The confrontation with a chronic disease or an injury includes the experience of physical symptoms and pain, aversive aspects of treatment, a stay in hospital away from home, and limitations on school or leisure activities. All this has the potential to cause adjustment problems such as anxiety, depression, posttraumatic stress disorder (PTSD), or behavioral problems. ${ }^{3}$ Pediatric patients use a broad repertoire of coping strategies. ${ }^{4}$ Emotion-focused strategies and cognitive strategies increase with age, reflecting the fact that children's cognitive capacities become more sophisticated. ${ }^{5}$ No single coping strategy can be effective for all types of stress relating to different points in time or contexts. ${ }^{6}$ Research findings show that pediatric patients prefer social support and avoidance strategies such as wishful thinking. ${ }^{3,7,8}$

Only a few studies have prospectively examined the associations between coping strategies and psychosocial adjustment in pediatric patients. In children with diabetes, avoidance strategies appear to be unfavorable with regard to social adjustment, ${ }^{9}$ school achievement and depressive symptoms. ${ }^{10}$ Frank et al. ${ }^{11}$ found an association between avoidance and anxiety and depression in children with cancer. On the other hand, successfully coping children use active coping, problem-solving and support seeking strategies more often. ${ }^{8}$ In sum, the evidence suggests that problem-focused coping and support seeking are related to better adjustment, whereas avoidant coping and self-blaming are associated with poorer adjustment. ${ }^{11}$ This conclusion agrees largely with the research findings on coping effectiveness in adults. ${ }^{12}$ However, the use of different assessment instruments, timeframes and different stressful and controllable situations contribute to heterogeneous findings. For example, as suggested by Stallard et al., ${ }^{7}$ timeframes may play a crucial role in terms of the effectiveness of coping: avoidant coping strategies may be adaptive in the first time after a stressor but ineffective in a longer term.

\section{Aim of the Study}

The aim of this study was twofold. First, we wanted to determine the prevalence of specific coping strategies and identify different 
indicators of psychosocial adjustment in a large group of recently hospitalized pediatric patients. Second, we aimed at studying the effectiveness of coping strategies with regard to psychosocial adjustment 1 year later.

\section{Methods}

\section{Participants}

Participants were assessed at four children's hospitals in the Germanspeaking part of Switzerland. Families were contacted consecutively within the first 2 weeks after their child's hospital admission and asked to participate in the study. All participants had to meet the following criteria: (1) hospitalization for at least 24 hours; (2) new diagnosis of cancer, type 1 diabetes, epilepsy or occurrence of an accident-related injury, excluding severe head injury; (3) age between 6 and 15 years; (4) fluency in German; (5) no previous evidence of intellectual impairment. During a period of 24 months, all children who met the criteria for inclusion in the study and their parents were contacted. Of 198 children meeting these criteria, 37 (28 boys, 9 girls) declined participation, so that the sample consisted of 161 children (response rate $81.3 \%$ ). There were no significant differences between participants and non-participants with regard to age and sex. Medical diagnosis differed in these two groups with a disproportionately higher number of injured patients among non-participants $\left(\chi^{2}=4.52, \mathrm{df}=1, p=0.03\right)$. Due to incomplete data at one of the two assessment points, the sample was reduced for some of the analyses. 138 children and 139 mothers provided complete data at T1, 128 children and 119 mothers at T2. In sum, 105 families generated a complete data record and 56 an incomplete record. Completers and non-completers did not differ with regard to age, sex, diagnosis and the baseline scores of the outcome variables, except higher internalizing CBCL scores in non-completers $(t=2.15, p=0.03)$.

\section{Measures}

Coping. Coping strategies were assessed using the How I Coped Under Pressure Scale (HICUPS), ${ }^{13}$ a multidimensional instrument that has been used in a variety of studies on coping in children from age 8 to 16 . The HICUPS is based on a four-factor coping model with the factors active coping, distraction, avoidance, and support seeking. It includes 45 items that are rated on a 4-point Likert scale ranging from 0 (never) to 3 (frequently). The present study used an authorized German translation, and the format was adapted to an interview version. Based on the authors' clinical experience and because religiousness seems to be associated with better psychological functioning in adults, ${ }^{14}$ a subscale comprising two items on religious coping was added. The two questions that were made up to constitute this scale are the following: (1) "Did you ask God for help?" (2) "Did you pray to God for comfort?" Some items of the distraction scale were excluded, because 
they describe physical activities that are not applicable with sick or convalescent children. Reliability coefficients for the basic factors in the current study corresponded to those reported by Ayers et al.: ${ }^{13}$ active coping $\alpha=0.87$; distraction $\alpha=0.62$; avoidance $\alpha=0.70$; seeking support $\alpha=0.76$; religious coping $\alpha=0.71$. Administration of the HICUPS entailed having the children generate one or several situations that had been difficult or stressful during their illness or injury experience. The interviewer gave some examples of such situations (e.g. to be in pain, long hospital stay, home sickness, to be absent from school), encompassing both physical and psychological stress, in order to stimulate the child's memory.

Child Post-Traumatic Stress Reactions. Child post-traumatic stress reactions were assessed by an interview version of the Child PTSD Reaction Index (RI). ${ }^{15}$ The RI is a widely used measure in childhood PTSD research. The scale is designed to asses post-traumatic stress reaction of school-age children and adolescents and contains 20 items that address children's fears about a traumatic event, symptoms relating to memories of the event, avoidance and general functioning. Responses reflect the frequency with which each symptom is experienced on a 5-point Likert scale ranging from 0 (none of the time) to 4 (most of the time). A total score is obtained by summing across all items after adjusting for reverse-scored items. Although the index does not provide a PTSD diagnosis according to DSM-IV, ${ }^{16}$ there is a scoring system that establishes a level of severity of PTSD. ${ }^{17}$ A total score of 12-24 indicates a mild level of PTSD; 25-39 a moderate level; 40-59 a severe level; and a score of $>60$ a very severe level. Scores above 24 are considered to be clinically relevant. The present study used a German version of the RI. ${ }^{18}$ In this study, the RI had a good internal consistency $(\alpha=0.77)$.

Behavioral Problems. Children's behavioral problems were assessed with the German version of the Child Behavior Checklist (CBCL) ${ }^{19,20}$ The CBCL is designed to record children's competencies and behavioral problems as reported by their parents. In this study, the questionnaire was completed by the mothers and the competencies section was not included. The 120 items are scored on a 3-point Likert scale ranging from not true (0) to often true (2). The CBCL contains eight problem syndrome scales as well as global scales for internalizing, externalizing and total problems. In this study, only the global scales were used. Internal consistency of the CBCL was found to be $\alpha=0.87$ for internalizing and $\alpha=0.91$ for externalizing problems.

Socio-Economic Status (SES). SES was calculated by means of a 6-point score of both paternal occupation and maternal education. The lowest SES score was 2 points, the highest 12 points. Three social classes were defined as follows: SES scores 2-5, lower class; SES scores 6-8, middle class; and SES scores 9-12, upper class. This measure was used in previous studies and was shown to be a reliable and valid indicator of SES in our community. ${ }^{4}$

Functional Status. Functional status with regard to daily physical activities was assessed by a single item 1 month and 1 year after the accident or 
diagnosis. The responsible physicians rated the functional status of patients using a 3-point Likert severity scale: $0=$ good functional status (no functional impairment), $1=$ moderate functional status (moderate impairment), $2=$ poor functional status (severe impairment). In order to increase interrater reliability, the different levels of functional impairment with regard to daily physical activities were defined in the questionnaire. This measure of functional status was used in a prior study and proved to be valid. ${ }^{4}$

Preceding Life Events. We assessed the occurrence of 12 major life events during the 12 months prior to the accident or diagnosis, such as change of residence, unemployment or parental separation, by obtaining mothers' reports. A life event score was computed by summing the number of life events for each family.

\section{Procedure}

The study was approved by the research ethics committees of all four hospitals. Written informed consent was obtained from parents in agreement with the children. Assessment was carried out 5-6 weeks after the child's admission to the hospital (T1) and at 1 year (T2). The children were assessed by means of a standardized interview conducted by trained graduate students of psychology. The interviews lasted from 30 to 60 minutes. Most interviews were conducted in the participants' homes; some were conducted at the hospital. Mothers were assessed at the same point of time using questionnaires. Demographic and medical variables were retrieved from the patients' records and the responsible physician.

\section{Statistical Analyses}

Chi-square analyses were used to compare nominal variables. $T$-tests, and ANOVAs were used for comparison of continuous variables. Pearson correlation coefficients were calculated to measure associations between sociodemographic variables, coping strategies, and adjustment scores. Bivariate correlation analyses were used to select predictor variables for the regression analyses. In all cases, $p<0.05$ was considered significant, and analyses were performed with two-sided tests. To examine variability of children's psychosocial adjustment after 1 year, hierarchical multivariate regression analysis was used. Psychosocial adjustment (T2) was the dependent criterion variable; personal characteristics, medical and social variables and coping strategies were independent predictors. Due to the high number of predictors, the more conservative adjusted $R^{2}$ statistic was used. Because the baseline value of a variable is usually the best predictor of the later score on the same variable, the baseline score (T1) of psychosocial adjustment was included in our regression analyses. To assess the effect of the coping strategies separately, they were included in the regression analyses in a second step after entering the personal, medical, social and baseline variables in a first step. The samples for the three different regression analyses contained those subjects who provided a complete data record. Kolmogorov-Smirnov Goodness of Fit Tests of the outcome variables showed normality for both CBCL scores, but not for the RI score. 
After square root transformation, this variable was normally distributed and used as the criterion variable in the multiple regression analysis.

\section{Results}

\section{Sample Characteristics}

Sample characteristics are presented in Table 1. Of the total of 161 participants, $62.7 \%$ (101) had accidental injuries and $37.2 \%$ (60) had a chronic disease. Twenty-five children were newly diagnosed with type 1 diabetes, 23 with cancer, and 12 with epilepsy. The injured children had experienced traffic accidents (58), leisure

Table 1

Characteristics of the Sample

\begin{tabular}{|c|c|c|c|c|c|c|}
\hline & $A l l$ & Injuries & Disease & $\chi^{2}$ & $t$ & $p$ \\
\hline$N$ & 161 & 101 & 60 & & & \\
\hline \multicolumn{7}{|l|}{ Sex } \\
\hline Female & 63 & 39 & 24 & 0.03 & & 0.86 \\
\hline Male & 98 & 62 & 36 & & & \\
\hline \multicolumn{7}{|l|}{ Age (years) } \\
\hline Mean $(S D)$ & $10.0(2.3)$ & $9.8(2.4)$ & $10.5(2.2)$ & & -1.94 & 0.05 \\
\hline \multicolumn{7}{|l|}{ SES } \\
\hline Lower & 14 & 9 & 5 & 7.94 & & 0.02 \\
\hline Middle & 94 & 50 & 44 & & & \\
\hline Upper & 42 & 33 & 9 & & & \\
\hline Unknown & 11 & 9 & 2 & & & \\
\hline \multicolumn{7}{|c|}{ Hospital stay (days) } \\
\hline Mean $(S D)$ & $10.7(9.4)$ & $8.4(8.1)$ & $14.6(10.3)$ & & -4.19 & $<0.001$ \\
\hline \multicolumn{7}{|c|}{ Functional Status at $\mathrm{T} 1$} \\
\hline Good $(0)$ & 79 & 51 & 28 & & & \\
\hline Moderate (1) & 60 & 37 & 23 & & & \\
\hline Poor (2) & 12 & 4 & 8 & & & \\
\hline Unknown & 10 & 9 & 1 & & & \\
\hline Mean $(S D)$ & $0.56(0.64)$ & $0.49(0.58)$ & $0.66(0.71)$ & & -1.62 & 0.11 \\
\hline \multicolumn{7}{|c|}{ Functional Status at T2 } \\
\hline Good (0) & 121 & 83 & 38 & & & \\
\hline Moderate (1) & 17 & 6 & 11 & & & \\
\hline Poor (2) & 1 & 0 & 1 & & & \\
\hline Unknown & 22 & 12 & 10 & & & \\
\hline Mean $(S D)$ & $0.14(0.37)$ & $0.07(0.25)$ & $0.26(0.49)$ & & -3.07 & 0.003 \\
\hline \multicolumn{7}{|l|}{ Life Events } \\
\hline Mean $(S D)$ & $1.21(1.36)$ & $1.12(1.17)$ & $1.38(1.61)$ & & -1.01 & 0.37 \\
\hline
\end{tabular}


activity or sports accidents (35), or burns (8). There were no differences among diagnostic groups with regard to sex, functional status at T1 and preceding life events. However, patients with chronic diseases were significantly older, had longer hospital stays, and were functionally more impaired at T2 than patients with accidents. SES was higher in the accident group. Over the period of eleven months the functional status increased significantly in the whole sample $(t=7.37, p<0.001)$.

\section{Prevalence of Coping Strategies}

Table 2 shows that the children used a great variety of coping strategies. Avoidance and distraction were used most frequently, i.e. by over three-quarter of the patients. Active and religious strategies were used by over the half of the children, whereas only around one-third of the sample reported the use of support seeking strategies. Notably, ranking and mean scores of the coping strategies did not differ between the two diagnostic groups. Analyses of variance showed no significant mean score differences of the coping strategy use between the subgroups diabetes, cancer, epilepsy, traffic accidents, leisure activity/sports accidents, and burns (active coping: $F=1.44, p=.21$; distraction: $F=0.32, p=0.90$; avoidance: $F=0.93, p=0.46$; support seeking: $F=0.14, p=0.98$; religious coping: $F=1.88, p=0.10$ ).

\section{Psychological Adjustment}

Psychological adjustment at T1 and T2 is shown in Table 3. Diagnostic groups did not differ significantly with regard to post-traumatic stress symptoms (PTSS). However at T1, behavioral problems as measured with the CBCL total score and the internalizing score were significantly more frequent in children with chronic diseases. At T2, this difference could be observed only with regard to the internalizing score. Over the period of 11 months the RI mean score did not change significantly. The proportion of children with a clinically relevant score $(>24)$ increased from $12.3 \%$ at $\mathrm{T} 1$ to $14.8 \%$ at T2. With regard to CBCL mean scores, only the internalizing score decreased significantly over time $(t=3.82, p<0.001)$. Analyses of variance concerning the adjustment scales (RI score, CBCL total score) showed no mean score differences between the subgroups of injuries. In the illness subgroups however, the RI scores at T2 differed significantly $(F=3.74, p=0.03)$ : Children with diabetes had a 


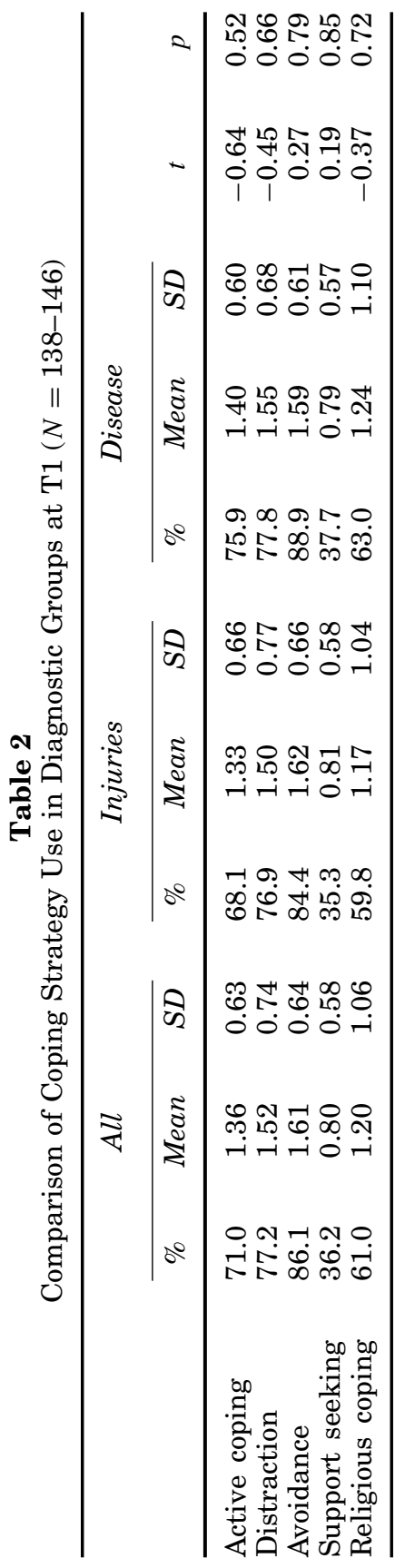


Table 3

Descriptive Statistics for Main Variables at T1 $(N=138-140)$ and $\mathrm{T} 2$ $(N=119-128)$

\begin{tabular}{lccccc}
\hline & All & Injuries & Disease & $t$ & $p$ \\
\hline RI at T1 $(N=138)$ & & & & & \\
$\quad$ Mean $(S D)$ & $12.7(9.4)$ & $13.8(9.9)$ & $10.7(8.1)$ & 1.90 & 0.06 \\
RI at T2 $(N=128)$ & $13.5(9.6)$ & $14.6(10.1)$ & $11.2(8.2)$ & 1.87 & 0.06 \\
$\quad$ Mean $(S D)$ & $55.6(10.0)$ & $53.7(8.9)$ & $58.6(10.9)$ & -2.88 & 0.01 \\
CBCL at T1 $(N=139-140)$ & & & & & \\
$\quad \begin{array}{l}\text { Mean Internalizing } \\
\quad \text { Score }(S D)\end{array}$ & $52.1(8.6)$ & $51.4(8.6)$ & $53.2(8.7)$ & -1.16 & 0.25 \\
$\quad \begin{array}{l}\text { Mean Externalizing } \\
\quad \text { Score }(S D)\end{array}$ & $54.7(9.6)$ & $53.3(9.3)$ & $56.8(9.8)$ & -2.16 & 0.03 \\
$\quad \begin{array}{l}\text { Mean Total Score }(S D) \\
\text { CBCL at T2 }(N=119)\end{array}$ & $52.9(11.0)$ & $50.8(10.3)$ & $55.7(11.3)$ & -2.35 & 0.02 \\
$\quad \begin{array}{l}\text { Mean Internalizing } \\
\quad \text { Score }(S D)\end{array}$ & & & & & \\
$\quad \begin{array}{l}\text { Mean Externalizing } \\
\quad \text { Score }(S D)\end{array}$ & $52.5(9.8)$ & $51.7(10.0)$ & $53.5(9.6)$ & -0.97 & 0.33 \\
$\quad$ Mean Total Score $(S D)$ & $53.8(10.5)$ & $52.1(10.0)$ & $56.0(10.9)$ & -1.90 & 0.06 \\
\hline
\end{tabular}

lower mean score (7.89) than children with cancer (12.75) or epilepsy (15.70). RI scores at T1 and CBCL total scores at $\mathrm{T} 1$ and $\mathrm{T} 2$ did not differ significantly.

\section{Associations of Predictor Variables with Psychosocial Adjustment}

Pearson correlations of sociodemographic, medical and coping variables (T1) with outcome variables (T2) are presented in Table 4. The sociodemographic and medical variables did not correlate with each other. Only the age of the child showed a low correlation with diagnosis: children with injuries were younger than those with chronic diseases.

The five coping factors correlated moderately with each other. Active coping, distraction and support seeking were associated with age: Older children used these strategies more often. Other sociodemographic variables were not associated with coping.

Table 4 also shows associations between a-priori predictors (T1) and outcome variables (T2). Diagnosis was associated with CBCL internalizing problems: children with chronic diseases had more internalizing problems. Moreover, preceding major life events were significantly correlated with higher scores on the CBCL internalizing and externalizing scales. 


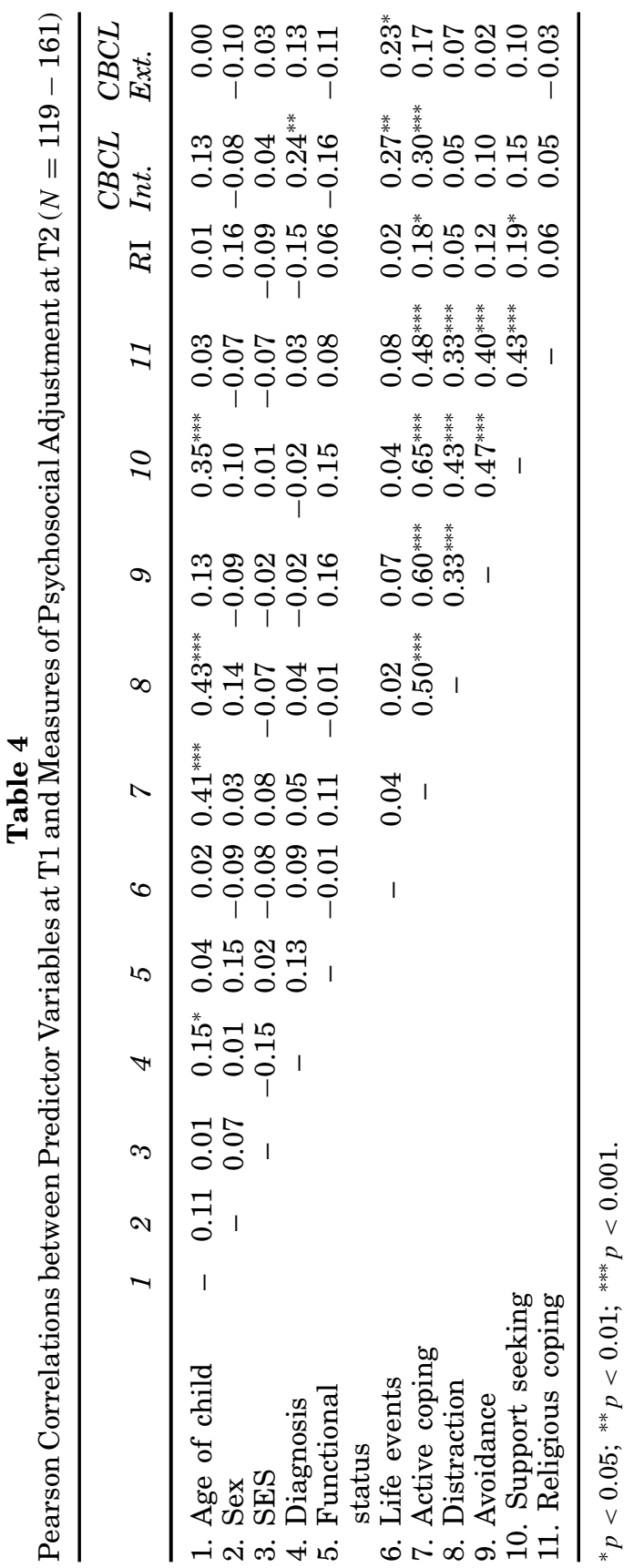


The use of active coping correlated significantly with two of the three outcome variables: children using more active coping strategies showed higher scores on PTSS and internalizing behavioral problems eleven months later. Also, children using support seeking strategies showed higher scores on PTSS at follow-up.

\section{Effects of Coping Strategies (T1) on Psychosocial Adjustment (T2)}

As the coping strategy use did not differ between the diagnostic groups, the multiple regression analyses were performed with the whole sample of pediatric patients. Results of multiple regression analysis with PTSS as the dependent variable are shown in Table 5. In sum, this model was able to explain $17 \%$ of the variance of the RI score. As expected, the RI baseline value was the best predictor of post-traumatic stress symptoms at T2. Religious coping was also found to be a significant predictor: children who used more religious coping reported fewer PTSD symptoms at 1 year.

Table 5 also shows the results of the multiple regression analyses with the T2 CBCL scales as the criterion variables. The highly significant models explained $48 \%$ and $52 \%$ of the CBCL score variances, respectively. Again, the symptomatology at T1 was the best predictor. Out of the five coping strategies, only active coping was found to be a significant predictor: children using active coping at $\mathrm{T} 1$ had higher scores on internalizing and externalizing problems at T2. Finally, functional status of the patients was a significant predictor: children who were functionally more impaired showed fewer internalizing behavior problems at $\mathrm{T} 2$.

\section{Discussion}

The results of this study show that pediatric patients with different conditions used a variety of specific coping strategies. This suggests that the children needed to adjust to different aspects of the stressful situation or that they were trying out a variety of strategies to deal with each aspect, or both. Avoidance and distraction were the most frequently used strategies. This is in line with findings by Ayers et al. ${ }^{21}$ on healthy children and with findings by Tyc et al. ${ }^{8}$ on pediatric cancer patients. Support seeking as a coping strategy was rarely used. This is consistent with earlier findings on healthy children. ${ }^{13}$ However, other researchers 


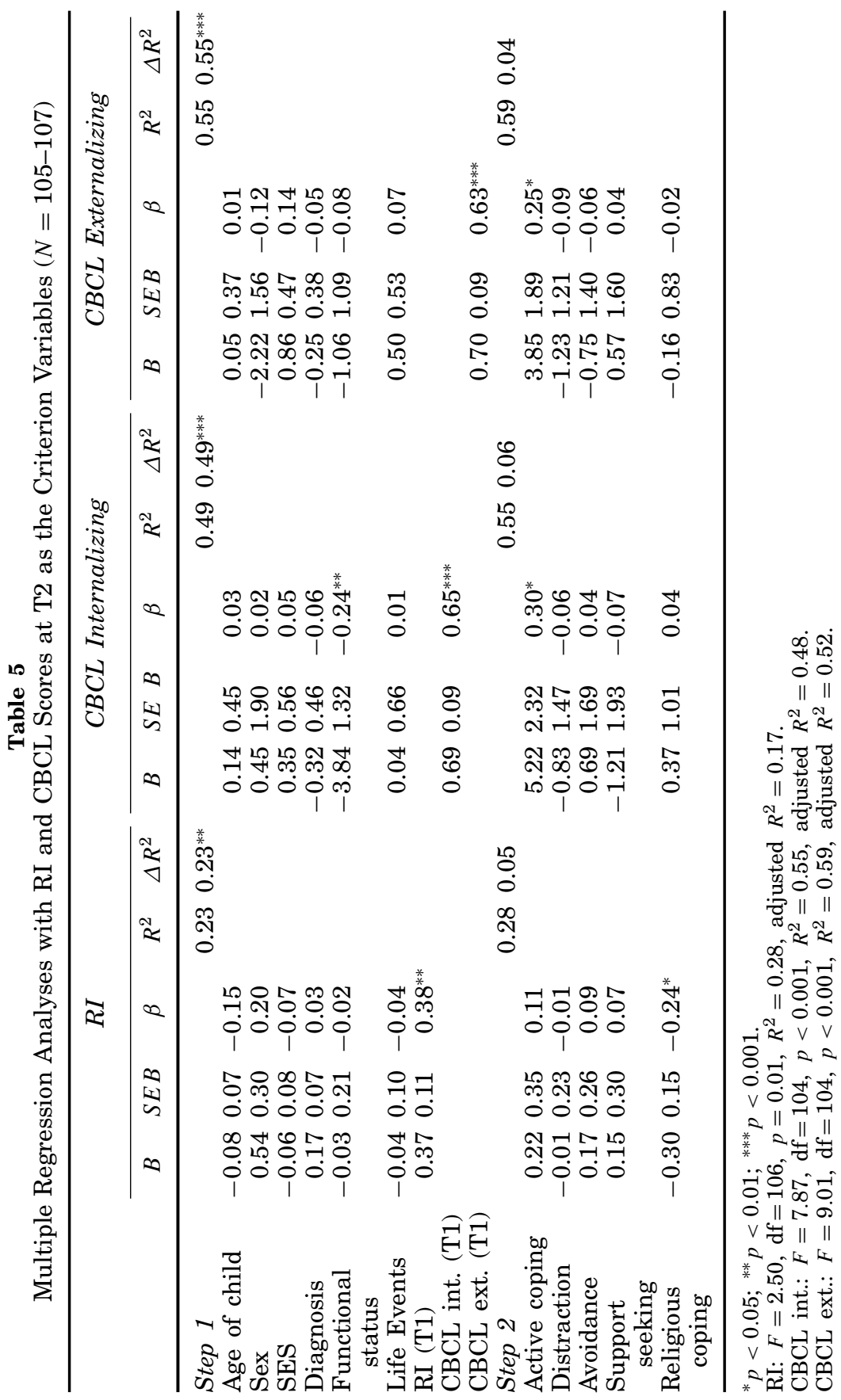


found the strategy of social support to play a more important role in pediatric populations. ${ }^{7}$ Notably, these researchers used the KID$\mathrm{COPE}^{22}$ to assess coping behavior. This instrument defines social support by the presence of individuals who provide support. In contrast, the HICUPS defines support as active seeking of emotional and instrumental support by the child. Active coping, distraction and support seeking were associated with age, reflecting the fact of growing cognitive and social abilities as described by Spirito et al. ${ }^{5}$

In this study, between $12 \%$ and $15 \%$ of the children had clinically relevant PTSS 1 month and 1 year post-hospitalization, respectively. These results are in line with other studies that found elevated prevalence rates of PTSS in pediatric patients. ${ }^{7,23}$ Also, children with a chronic disease had significantly more internalizing behavioral problems than children with injuries. Compared to a German population of healthy children, ${ }^{20}$ chronically ill children showed a significant increase of CBCL scores both at 1 month and 1 year. Children with injuries showed increased scores on the internalizing scale only at 1 month. These findings are consistent with other longitudinal studies showing that in the initial period after a diagnosis or an injury, feelings of loneliness, sadness and anxiety are common. ${ }^{24} \mathrm{RI}$ scores, CBCL externalizing and CBCL total scores did not change significantly from $\mathrm{T} 1$ to $\mathrm{T} 2$. Only the CBCL internalizing mean score decreased significantly. This positive change over time can be observed in both, injured and ill children. We assume that a commonly used psychological intervention program for children with cancer can help to cope with feelings of sadness and anxiety. Conversely, for the children of all other diagnostic subgroups, psychological support is not systematically provided. One may speculate that feelings of anxiety and sadness as a consequence of an accident or a chronic disease decrease over time whereas post-traumatic stress symptoms do not alter.

As to the effectiveness of specific coping strategies, our findings were unexpected. Problem-focused and support seeking coping did not predict a better psychosocial adjustment. In addition, active coping was associated with higher levels of PTSS, internalizing and externalizing behavioral problems. This is in contradiction to findings among adults. Possibly, this may be explained by differences between children's and adults' coping (e.g., cognitive development). Aldwin and Revenson ${ }^{25}$ suggested a reciprocal relation between adjustment and coping, where active coping affects psychosocial adjustment, but psychosocial adjustment in turn activates coping. Another explanation relates to the time interval between the 
two assessment points in our study. Possibly, active coping strategies only have short-time positive effects on adjustment that fade 11 months later. Finally, characteristics of the situation also influence coping effectiveness. It has been suggested that active coping strategies could be maladaptive in uncontrollable situations, such as being treated in a hospital. ${ }^{26}$ Lazarus and Folkman ${ }^{1}$ note that problem-focused strategies are only effective in controllable situations, whereas emotion regulative strategies are adaptive in uncontrollable situations.

We found no influences of distraction and avoidance on psychosocial adjustment. This is in line with several earlier studies of children with diabetes ${ }^{9,10}$ and cancer. ${ }^{11}$ Interestingly, avoidant and distraction strategies may also have positive adjustment effects in a short-term perspective. ${ }^{12}$ The children in this sample used these strategies most frequently. Nevertheless, no effects on psychosocial adjustment could be detected eleven months later. Also, support seeking had no influence on psychosocial adjustment. This does not correspond with earlier findings by Tyc et al., ${ }^{8}$ who found positive effects of social support seeking in pediatric patients. Interestingly, in contrast to these negative findings, religious coping had an effect on PTSS: children using more religious coping reported fewer symptoms after 1 year. A comparison of this finding with other studies is not possible, because religious coping has not been examined in children to date. Possibly, praying to God functions like a cognitive monologue that enhances hope and reduces feelings of loneliness. Another possible explanation might include the fact that religious coping is associated with other distal factors such as family functioning which in turn may either moderate or mediate psychosocial adjustment. Further research is needed to examine the mechanisms by which religious coping contributes to better adjustment.

\section{Limitations}

Some limitations of this study need to be addressed. First, the HICUPS was not originally designed to assess varying coping responses in disease, injury, or treatment-related situations, and the validity of the German version of this questionnaire has not yet been demonstrated. Moreover, some of the coping subscales, in particular the distraction scale, had rather low reliability coefficients and nothing is known about appropriateness of the HICUPS for children as young as 6 years of age. However, reliability of most of the scales was satisfactory and did not improve when only children above the age of 10 were 
included in a separate analysis. Second, we cannot explain the facts that children who were functionally less impaired and participants with incomplete data had higher internalizing CBCL scores. Third, our sample is quite heterogeneous and children differed in some of the psychosocial and medical variables. Although mean scores of coping did not differ between the two diagnostic groups and the diagnosis was not predictive of adjustment, conditions might still be too different to interpret the results as characteristics of one specific pediatric group. A fourth limitation refers to our working model for measuring coping effectiveness. The time lag of 11 months between the assessment of coping and psychosocial adjustment is long compared to other studies. $^{7,21}$ The particular stressors and the overall stress level influencing coping and adjustment, change uncontrollably over time and predefined time lags may miss relevant new stressors. We know little about how the effects of coping on adjustment may vary over time. It should be acknowledged that coping strategies can change over time and as the demands of the situations change, certain strategies might be useful in the early days but less useful as the situations changes. Fifth, causal relations between coping and adjustment were tested only as far as coping affects adjustment. However, prior levels of adjustment, for example in the first month after diagnosis or accident, may have affected the use of coping strategies as well. Finally, our study did not assess potential mediators or moderators concerning the family functioning that could help to test hypotheses relating to the positive effects of religious coping.

Despite these limitations, the present study has several strengths, including its longitudinal design, its prospective collection of data, the highly standardized assessment instruments, and its careful statistical analyses.

\section{Implications}

The results of this study suggest some possible issues for future research. To investigate the effect of coping on adjustment, longitudinal studies are the best choice. However, the effects of coping might better be tested at several time intervals. This would provide information about the course of adjustment over time. Also, assessment points might be adapted to the course of the illness or the healing process instead of following a fixed time schedule. One of the surprising results of our study was the positive effect of religious coping strategies on post-traumatic stress symptoms. As little is known about religion as a coping strategy, more research in this area might 
be worthwhile. Further research on the role of active coping and its effect on adjustment is also required, since the results of this study contradict the general idea that active coping is an effective and helpful strategy in ill or injured children.

There are also some clinical implications that can be drawn from this study. Our findings confirm the need for careful evaluation of psychological parameters in pediatric patients. ${ }^{27}$ Particularly, injured children may need specific attention regarding PTSS. This is important in view of the finding that initial psychological problems predict later psychological problems. A short psychological and behavioral screening of pediatric children as suggested by Stallard et al. ${ }^{28}$ could help to identify those patients with a greater risk, and timely support could be offered.

\section{Summary}

The current paper examined the prevalence of coping strategy use in 161 pediatric patients and the effectiveness of coping strategies with regard to psychosocial adjustment 1 year later. The results of this study showed that pediatric patients with different conditions used a variety of specific coping strategies, but contradicted the idea that active coping is in general an effective strategy. Support seeking strategies, distraction, and avoidance had no impact on long-term psychosocial adjustment and religious coping reduced post-traumatic stress symptoms. The association of coping strategies and psychosocial adjustment is complex and must be investigated from a transactional point of view. Further longitudinal research is needed for a better comprehension of the short- and long-term effects and to explain possible differences between children's and adults' coping.

The findings that initial psychological problems predict the longterm symptomatology confirmed the need for careful evaluation of psychological parameters in pediatric patients. Newly diagnosed or injured pediatric patients deserve special attention, because of their elevated and persistent rates of post-traumatic stress reactions and behavioral problems.

\section{Acknowledgments}

This research was funded by grants from the Swiss Research Foundation Child and Cancer, the Gebert Ruef Foundation, the Hugo and Elsa Isler Foundation, the Anna Mueller Grocholski Foun- 
dation and Bayer Diagnostics. We thank the families who participated in this project as well as Felix H. Sennhauser, Karin Ribi, Hanspeter E. Gnehm, Karin Timm, and Joseph Laimbacher for their contributions to this project.

\section{References}

1. Lazarus RS, Folkman S: Stress, Appraisal, and Coping. New York: Springer, 1984.

2. Rudolph KD, Dennig MD, Weisz JR: Determinants and consequences of children's coping in the medical setting: Conceptualization, review, and critique. Psychol Bull 118: 328-357, 1995.

3. Wallander JL, Varni JW: In: Stress and Coping in Child Health, eds. Greca AML, Siegel LJ, Wallander JL, Walker CE. New York: The Guilford Press, 1992, pp. 279-298.

4. Landolt MA, Vollrath M, Ribi K: Predictors of coping strategy selection in paediatric patients. Acta Paediatr 91: 954-960, 2002.

5. Spirito A, Stark LJ, Tyc VL: Stressors and coping strategies described during hospitalization by chronically ill children. J Clin Child Psychol 23: 314-322, 1994.

6. Compas BE: Coping with stress during childhood and adolescence. Psychol Bull 101: 393-403, 1987.

7. Stallard P, Velleman R, Langsford J, Baldwin S: Coping and psychological distress in children involved in road traffic accidents. $\mathrm{Br} J$ Clin Psychol 40: 197-208, 2001.

8. Tyc VL, Mulhern RK, Jayawardene D, Fairclough D: Chemotherapy-induced nausea and emesis in pediatric cancer patients: An analysis of coping strategies. J Pain Symptom Manage 10: 338-347, 1995.

9. Grey M, Cameron ME, Thurber FW: Coping and adaptation in children with diabetes. Nurs Res 40: 144-149, 1991.

10. Reid JR, Dubow EF, Carey TC: Developmental and situational differences in coping among children and adolescents with diabetes. J Appl Dev Psychol 16: 529-554, 1995.

11. Frank NC, Blount RL, Brown RT: Attributions, coping, and adjustment in children with cancer. J Pediatr Psychol 22: 563-576, 1997.

12. Suls J, Fletcher B: The relative efficacy of avoidant and nonavoidant coping strategies: A meta-analysis. Health Psychol 4: 249-288, 1985.

13. Ayers TS, Sandler IN, West SG, Roosa MW: A dispositional and situational assessment of children's coping: Testing alternative models of coping. J Pers 64: 923-958, 1996.

14. Diener E, Suh EM, Lucas RE, Smith HL: Subjective well-being: Three decades of progress. Psychol Bull 125: 276-302, 1999.

15. Frederick CJ, Pynoos R, Nader K: Childhood Posttraumatic Stress Reaction Index-A Copyrighted Instrument. Los Angeles: University of California, 1992.

16. American Psychiatric Association: Diagnostic and Statistical Manual of Mental Disorders, 4th ed. Washington, DC: Author, 1994.

17. Pynoos RS, Goenjian A, Tashjain M, et al.: Post-traumatic stress reaction in children after the 1988 Armenian earthquake. Br J Psychiatr 163: 239-247, 1993.

18. Landolt MA, Vollrath M, Ribi K, Gnehm HE, Sennhauser FH: Incidence and associations of child and parental posttraumatic stress symptoms in pediatric patients. J Child Psychol Psychiatr 44: 1199-1207, 2003. 
19. Achenbach TM: Manual for the Child Behavior Checklist 4-18 and 1991 Profile. Burlington, VT: University of Vermont, 1991.

20. Doepfner M, Plück J, Bölte S, Lenz K, Melchers P, Heim K: Elternfragebogen über das Verhalten von Kindern und Jugendlichen. Deutsche Bearbeitung der Child Behavior Checklist (CBCL/4-18). Köln: KJFD, 1998.

21. Sandler IN, Tein JY, West SG: Coping, stress, and the psychological symptoms of children of divorce: A cross-sectional and longitudinal study. Child Dev 65: 1744-1763, 1994.

22. Spirito A, Stark LJ, Williams C: Development of a brief coping checklist for use with pediatric populations. J Pediatr Psychol 13: 555-574, 1988.

23. Kazak AE, Barakat LP, Meeske K, et al.: Posttraumatic stress, family functioning and social support in survivors of childhood cancers. J Am Acad Child Adolesc Psychiatr 37: 823-831, 1997.

24. Boekaerts M, Roeder I: Stress, coping and adjustment in children with a chronic disease: A review of the literature. Disabil Rehabil 21: 311-337, 1999.

25. Aldwin CM, Revenson TA: Does coping help? A reexamination of the relation between coping and mental health. J Pers and Soc Psychol 53: 337-348, 1987.

26. Weisz JR, McCabe MA, Denning MD: Primary and secondary control among children undergoing medical procedures: Adjustment as a function of coping style. J Consult Clin Psychol 62: 324-332, 1994.

27. Landolt MA, Vollrath M, Ribi K, Timm K, Sennhauser FH, Gnehm HE: Inzidenz und Verlauf posttraumatischer Belastungsreaktionen nach Verkehrsunfällen im Kindesalter. Kindheit und Entwicklung 12: 184-192, 2003.

28. Stallard P, Velleman R, Baldwin S: Psychological screening of children for post-traumatic stress disorder. J Child Psychol Psychiatr 40: 1075-1082, 1999. 